\title{
The Nuclei of Nearby Radio-Loud Ellipticals
}

\author{
G. A. Verdoes Kleijn, P. T. de Zeeuw \\ Leiden Observatory, Postbus 9513, 2300 RA, Leiden, The Netherlands
}

S. A. Baum, C. P. O'Dea, R. P. van der Marel, C. Xu

Space Telescope Science Institute, 3700 San Martin Drive, Baltimore, $M D$ 21218, USA

C. M. Carollo, J. Noel-Storr

Columbia University, Dept. of Astronomy, New York, NY 10027, USA

\begin{abstract}
We have observed a complete sample of 21 nearby $(D<$ $70 h^{-1} \mathrm{Mpc}$ ) Fanaroff \& Riley Type I galaxies with HST/WFPC2 and detected dust disks and lanes in 19 of them. The radio jets are roughly perpendicular to the dust which is used to constrain the Doppler boosting factors of the radio jet and cores. The VLBA core flux correlates with the central $\mathrm{H} \alpha+[\mathrm{NII}]$ flux which might indicate that the VLBA core is dominated by an isotropic component. Twelve galaxies show nuclear optical sources. We discuss various possible origins for this emission.
\end{abstract}

\section{Introduction}

The Fanaroff \& Riley Type I (FR I) radio galaxies in the nearby $(z<0.03)$ universe can be characterized as early-type galaxies with jets emanating from an AGN which is powered by a black hole (BH). These AGN display only weak nuclear optical line and continuum emission. The FR I stellar hosts and their unresolved cores bear resemblance to both normal early-type galaxies with radio cores, which constitute a considerable fraction of the nearby early-type population ( $\sim 50 \%$ at $M_{B}=-22$, cf. Sadler 1997) and to early-type galaxies with unresolved blue optical spikes (Carollo et al. 1997). On the other hand, the FR I galaxies appear to be scaled-down versions of powerful radio galaxies and quasars in the distant universe which have strong nuclear optical line and continuum emission. For a physical understanding of the connection between active and normal galaxies, it is important to determine how these low-luminosity active nuclei and their jets form and evolve. Relevant questions are then: where did the accreted matter come from, how does this accretion trigger jet formation, and what is the origin of the optical AGN luminosity? We are using HST to study the centers of a complete sample of FR I radio galaxies and to isolate weak optical nuclear activity from the stellar background (Verdoes Kleijn et al. 1999). Here we discuss further interpretation of the WFPC2 data in combination with $\mathrm{VLBA}$ radio data presented by $\mathrm{Xu}$ et al. (2000). 

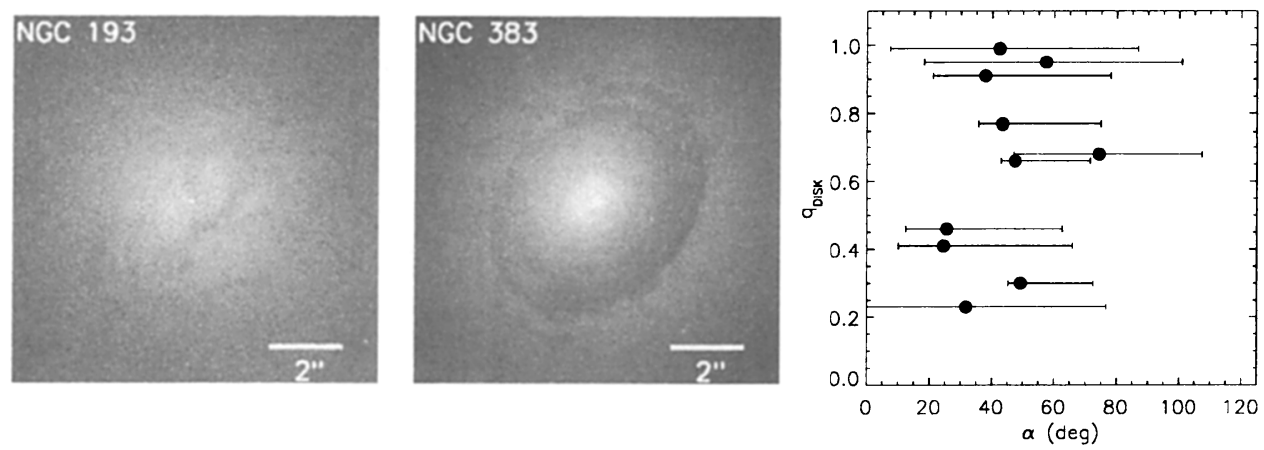

Figure 1. Left and middle: $V$-band images of two sample galaxies. NGC 193 has a dust lane and NGC 383 has a dust disk. Both galaxies have a blue nuclear optical source. Right: the axis ratio of the dust disk plotted versus the range of the angle $\alpha$ between the sides of the dust disk symmetry axis and the radio-jet axis, that are closest to the direction of the line of sight. Horizontal bars indicate the allowed range for $\alpha$ and the solid dots indicate the median $\alpha$.

\section{Orientation of Dust and Radio Jets}

We detected dust in the centres of 19 galaxies (Fig. 1). The dust extent ranges from a few hundred pc to a few kpc. In eleven galaxies, the dust morphology is smooth and elliptical (a 'disk'), while in eight galaxies it is filamentary with wisps and bends (a 'lane'). Lanes are roughly perpendicular to the radio jets: the position angle difference $\triangle \mathrm{PA}$ is in the range $68^{\circ}-90^{\circ}$. Processes that cause such a preferred orientation are discussed in e.g., Quillen \& Bower (1999). By contrast, all disks closely align with the galaxy major axis. The $\Delta \mathrm{PA}$ of the disk major axis and radio jet is in the range range $23^{\circ}-90^{\circ}$. One can assume that (i) dust disks are circular and (ii) the brightest side of the radio jet is approaching the observer. The allowed range of the intrinsic angle $\alpha$ between the sides of both the dust disk symmetry axis and the radio jet axis that are closest to the direction of the line of sight, can then be computed. By definition, the radio jet inclination is in the range $0^{\circ}-90^{\circ}$, but $\alpha$ can take a value in the range $0^{\circ}-180^{\circ}$. The right panel in Fig. 1 shows that the allowed range of $\alpha$ for each dust disk is constrained such that the dust disk symmetry axis and the radio jet axis tend to 'align' (i.e., $\alpha<90^{\circ}$ ). Further analysis indicates that the angle with the line of sight for jet and disk are expected to differ typically by only $\sim 10^{\circ}-20^{\circ}$. A similar result was derived by Capetti \& Celotti (1999) for a small pilot sample.

\section{Doppler Boosting of Radio Jets and Cores}

Xu et al. (2000) report the surface brightness ratio $S$ of VLBA jet and counter jet at $1670 \mathrm{MHz}$ for the sample galaxies. If the jets are intrinsically symmetric and ejected in opposite directions, $S$ depends on jet inclination and velocity (e.g., Urry \& Padovani 1995). As discussed in the previous section, the dust disk inclination is a reasonable estimator for the jet inclination and can constrain the 

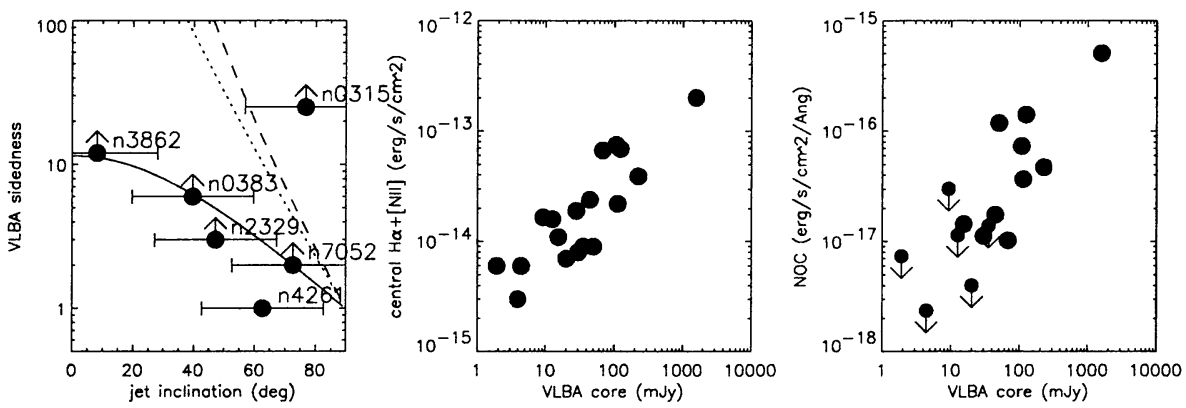

Figure 2. Left: the VLBA jet to counter jet surface brightness ratio $S$ versus dust disk inclination. The arrows indicate lower limits. The curves describe $S$ for an isotropic continous jet with no preferred direction of the magnetic field, a radio spectral index $\alpha=0.75$ and $\gamma=1.1$ (solid line), $\gamma=2.0$ (dotted line) and $\gamma=10.0$ (dashed line). The horizontal error bars indicate the uncertainty in the jet inclination. Middle: $\mathrm{H} \alpha+[\mathrm{NII}]$ flux in the central $1^{\prime \prime}$ versus VLBA core flux (1670 $\mathrm{MHz}$ ). Right: flux of the nuclear optical sources ( $I$-band) detections and upper limits versus VLBA core flux.

jet velocity. Fig. 2 plots $S$ versus jet inclination together with model predictions for constant jet velocity $v$, assuming an isotropic continuous jet with no preferred direction of the magnetic field and a radio spectral index $\alpha=0.75\left(f_{\nu} \sim \nu^{-\alpha}\right)$ typical for jets. The observed values of $S$ cannot constrain $v$ very well given that most are lower limits (i.e., the counter jet is not observed) and given the $\sim 20^{\circ}$ uncertainty of the jet inclination. For NGC 315 we can constrain the Lorentz factor to be $\gamma>10$.

The VLBA radio-core flux (unresolved at the parsec scale) correlates tightly with central $\mathrm{H} \alpha+[\mathrm{NII}]$ flux (Fig. 2). Interestingly, a similar correlation is found for radio-core galaxies (Ho 1999). If the $\mathrm{H} \alpha+[\mathrm{NII}]$ flux is emitted isotropically and correlates tightly with intrinsic VLBA core flux, the observed scatter in the correlation might be due to Doppler boosting. The low scatter in the correlation then constrains $\gamma<2$. Indeed, no dependence of the VLBA core flux on dust disk inclination is found. Thus it seems that the VLBA core flux is dominated by a relatively isotropic component instead of a relativistic jet. This isotropic component might have uncollimated relativistic motion.

\section{Nuclear Optical Sources}

Twelve galaxies show blue nuclear optical sources (NOS) unresolved with WFPC2, corresponding to sizes of tens of parsec or less. The right panel in Fig. 2 shows NOS flux versus VLBA radio core flux. The observed correlation is significant at the $99.999 \%$ level (generalized Kendall's Tau test). This agrees with results by Chiaberge, Capetti, \& Celotti (1999). Radio core emission is generally assumed to be self-absorped synchrotron emission. The correlation might indicate that the NOS is also synchrotron emission. The radio-to-optical spectral index varies 
between 0.43 and 0.85 . These values are consistent with those found for galaxies in our sample with extended optical jets: 3C 66B, 3C 31 and M87 (Butcher, van Breugel \& Miley 1980; Biretta, Stern, \& Harris 1991). The slope of the log-log correlation, $s=1.04 \pm 0.24$, although not well determined, is consistent with a power-law spectral energy distribution (SED) from radio to optical wavelengths.

Alternatively, the NOS might be emission from the accretion disk and/or flow. For example, Di Matteo et al. (2000) obtain a reasonable fit to the nuclear radio to X-ray SED of M87 using an ADAF model with matter outflow. However, the models that fit the observed X-ray SED underpredict the nuclear optical emission by a factor of $\sim 4$. Furthermore, Di Matteo et al. note evidence for a contribution to the flux by the synchrotron jet at radio and millimeter fluxes. If the accretion disk is inside an optically thick torus, the NOS detection rate implies an opening angle $\sim 130^{\circ}$. Broad emission-line regions are commonly detected in powerful AGN but typically not detected in FR Is. The large opening angle would then suggest BLRs are generally not present in FR I galaxies

Finally, the NOS might be produced by a nuclear starburst. The high NOS detection rate would require a continuous starburst on time scales on the order of the radio-source lifetime, typically estimated to be $10^{7-8} \mathrm{yr}$ (cf. Chiaberge, Capetti, \& Celotti 1999). However, detailed studies of the optical nuclear spectra of M87 and M84 indicate their NOS are indeed not produced by a starburst but by non-thermal AGN emission ( Kormendy 1992; van der Marel 1994; Bower et al. 2000).

\section{References}

Biretta, J.A., Stern, C.P., \& Harris, D.E. 1991, AJ, 101, 1632

Bower, G.A., et al. 2000, ApJ, 534, 189

Butcher, H.R., van Breugel, W., \& Miley, G.K. 1980, ApJ, 235, 749

Capetti, A., \& Celotti, A. 1999, MNRAS, 304, 434

Carollo, C.M., Franx, M., Illingworth, G.D., \& Forbes, D.A. 1997 ApJ 481, 710

Chiaberge, M., Capetti, A., \& Celotti, A. 1999, A\&A, 349, 77

Di Matteo, T., Quataert, E., Allen, S.W., Narayan, R., \& Fabian, A.C. 2000, MNRAS, 311, 507

Ho, L.C. 1999, ApJ, 510, 631

Kormendy, J. 1992, ApJ, 388, L9

Quillen, A.C., Bower, G.A. 1999, ApJ, 522, 718

Sadler, E.M. 1997, in ASP Conf. Ser. Vol. 115, The Nature of Elliptical Galaxies, ed. M. Arnaboldi, G.S. Da Costa, \& P. Saha (San Fransisco: ASP), 411

Urry, C.M., Padovani, P. 1995, PASP, 107, 803

van der Marel, R.P. 1994, MNRAS, 270, 271

Verdoes Kleijn, G.A., Baum, S.A., de Zeeuw, P.T., O'Dea, C.P. 1999, AJ, 118, 2592

Xu, C., Baum, S.A., O'Dea, C.P., Wrobel, J.M., \& Condon, J.J. 2000, AJ, submitted (astro-ph/0009124) 